\title{
ON A CLASS OF PARTICULAR SOLUTIONS OF THE PROBLEM OF FOUR BODIES*
}

BY

\author{
FOREST RAY MOULTON
}

Introduction. In 1772 LAGRANGe published a celebrated memoir on the Problem of. Three Bodies, which contained all the solutions in which the ratios of the mutual distances of the bodies are constants. He found two distinct configurations. In the one, the three bodies always lie in a straight line; in the other, they are always at the vertices of an equilateral triangle. Their distribution upon the line depends upon their masses, being explicitly defined by the real positive root of a certain quintic equation. The equilateral triangular configuration is possible for all distributions of the masses. In both cases the three bodies move in the same plane, in conic sections with respect to each other or with respect to their common center of gravity, and in such a manner that the law of areas is true for each body considered separately.

No other periodic solutions of the motion of three or more bodies were discovered for more than a century, although many splendid papers appeared on the Problem of Three Bodies. A new impetus was given to the subject by the celebrated memoir of Dr. HILL on the Lunar Theory, $\dagger$ in which he discussed a new species of periodic solutions. He made the restrictions that one body should be infinitesimal, and that the finite bodies should describe circles around their center of gravity. For the purposes of the applications to the Lunar Theory he neglected the ratio of the distances of the moon and the sun, thus introducing errors in the numerical results which in certain cases became important.

Professor DARwin has more recently taken up the subject $\ddagger$ by a method not essentially different from that employed by HrLL, and discussed a great many orbits in detail, not neglecting the solar parallax. He has considered only cases in which the infinitesimal body moves in the plane of the finite bodies with direct motion.

To these must be added the masterful researches $\S$ of PoIncare, who has

\footnotetext{
* Presented to the Society at the Columbus meeting, Aug. 25, 1899, under a slightly different title. Received for publication Aug. 25, 1899.

† American Journal of Mathematics, vol. 1.

† Acta Mathematica, vol. 21.

is Les Méthodes Nouvelles de la Mécanique Céleste, I and III.
}

(Trans. Am. Math. Soc., 2.) 
proved the existence of several classes of infinite numbers of periodic solutions of the Problems of Three Bodies. His results are not given explicitly, and not all of the circumstances of the motion are known as they are in the previous cases.

This paper treats of the Problem of Four Bodies, three of which are finite, moving in circles according to one or the other of the solutions of LAGRANGE, while the fourth is infinitesimal. The motion of the systems is referred to axes rotating with uniform angular velocity. An integral, similar to that of J ACOBI in the Problem of Three Bodies, is found, which leads to the definition of the surfaces of zero relative velocity. For certain initial conditions these surfaces have double points, all of which are found.

It is shown that if the infinitesimal body be started at any double point of the surfaces, with zero relative velocity, it will always remain in the same position with respect to the finite bodies. Therefore they are particular solutions of the motion of the system, and it is true that they are the only ones in which the mutual distances of the bodies are constants. There are eighteen solutions of arbitrary periods of revolution in which the finite bodies lie in a line, and ten in which they are at the vertices of an equilateral triangle.

\section{§1. The differential equations of motion.}

Suppose the masses of the finite bodies are $m_{1}, m_{2}$, and $m_{3}$ respectively. Suppose the origin is taken at the center of gravity of the system. Let the coördinates of $m_{i}$ be $\xi_{i}, \eta_{i}, \zeta_{i}, i=1,2,3$, and of the infinitesimal body, $\xi$, $\eta$, and $\zeta$. Suppose the units are chosen so that the Gaussian constant is equal to unity. Let

and

$$
\rho_{i}=\sqrt{\left(\bar{\xi}-\xi_{i}\right)^{2}+\left(\eta-\eta_{i}\right)^{2}+\left(\zeta-\zeta_{i}\right)^{2}} \quad(i=1,2,3)
$$

$$
U=\frac{m_{1}}{\rho_{1}}+\frac{m_{2}}{\rho_{2}}+\frac{m_{3}}{\rho_{3}}
$$

Then the differential equations of motion of the infinitesimal body are

$$
\left\{\begin{array}{l}
\frac{d^{2} \xi}{d t^{2}}=\frac{\partial U}{\partial \xi}, \\
\frac{d^{2} \eta}{d t^{2}}=\frac{\partial U}{\partial \eta} \\
\frac{d^{2} \zeta}{d t^{2}}=\frac{\partial U}{\partial \zeta}
\end{array} .\right.
$$

The motion of the system is referred to $x y$-axes rotating in the $\xi r_{\text {-plane }}$ by the substitution 


$$
\left\{\begin{array}{l}
\xi_{i}=x_{i} \cos \theta-y_{i} \sin \theta, \\
\eta_{i}=x_{i} \sin \theta+y_{i} \cos \theta, \\
\zeta_{i}=z_{i},
\end{array}\right.
$$

where $\theta$ is a function of the time.

Equation (1) is transformed by this substitution into

(8)

$$
\left\{\begin{aligned}
\frac{d^{2} x}{d t^{2}}-2 \frac{d \theta}{d t} \frac{d y}{d t}-\left(\frac{d \theta}{d t}\right)^{2} x-\frac{d^{2} \theta}{d t^{2}} y & =\frac{\partial U}{\partial x} \\
\frac{d^{2} y}{d t^{2}}+2 \frac{d \theta}{d t} \frac{d x}{d t}-\left(\frac{d \theta}{d t}\right)^{2} y+\frac{d^{2} \theta}{d t^{2}} x & =\frac{\partial U}{\partial y} \\
\frac{d^{2} z}{d t^{2}} & =\frac{\partial U}{\partial z}
\end{aligned}\right.
$$

If the axes rotate with the uniform angular velocity, $n$, the system (3) becomes

(4)

$$
\text { (4) }\left\{\begin{aligned}
\frac{d^{2} x}{d t^{2}}-2 n \frac{d y}{d t}=n^{2} x+\frac{\partial U}{\partial x}=n^{2} x & -\frac{m_{1}\left(x-x_{1}\right)}{\rho_{1}^{3}} \\
\frac{d^{2} y}{d t^{2}}+2 n \frac{d x}{d \bar{t}} & =n^{2} y+\frac{m_{2}\left(x-x_{2}\right)}{\rho_{2}^{3}}-\frac{m_{3}\left(x-x_{3}\right)}{\rho_{3}^{3}}=n^{2} y-\frac{m_{1}\left(y-y_{1}\right)}{\rho_{1}^{3}} \\
-\frac{m_{2}\left(y-y_{2}\right)}{\rho_{2}^{3}}-\frac{m_{3}\left(y-y_{3}\right)}{\rho_{3}^{3}} & -\frac{m_{2}\left(z-z_{2}\right)}{\rho_{2}^{3}}-\frac{m_{3}\left(z-z_{3}\right)}{\rho_{3}^{3}} .
\end{aligned}\right.
$$

The substitution

(5)

$$
\left\{\begin{array}{c}
\frac{d x}{d t}-n y=x^{\prime}, \\
\frac{d y}{d t}+n x=y^{\prime}, \\
\frac{d z}{d t}=z^{\prime}, \\
H=\frac{1}{2}\left(x^{\prime}+n y\right)^{2}+\frac{1}{2}\left(y^{\prime}-n x\right)^{2}+z^{\prime 2}-\frac{1}{2} n^{2}\left(x^{2}+y^{2}\right)-U
\end{array}\right.
$$

transforms the system (4) into the canonical system 


$$
\begin{cases}d x=\frac{\partial H}{\partial x^{\prime}}, & \frac{d x^{\prime}}{d t}=-\frac{\partial H}{\partial x}, \\ d y=\frac{\partial H}{\partial y^{\prime}}, & \frac{d y^{\prime}}{d t}=-\frac{\partial H}{\partial y}, \\ d z=\frac{\partial H}{\partial t^{-}}, & \frac{d z^{\prime}}{d t}=-\frac{\partial H}{\partial z}\end{cases}
$$

§ 2. Integrals of the differential equations.

Suppose the angular velocity of revolution of the finite bodies, which we now assume move in circles according to one or the other of the Lagrangian solutions, is also $n$; then $x_{i}, y_{i}, z_{i}$ are constants. Therefore, from system (6) there results. the integral $H+C=0$, or, in full,

$$
\left(\frac{d x}{d t}\right)^{2}+\left(\frac{d y}{d t}\right)^{2}+\left(\frac{d z}{d t}\right)^{2}=n^{2}\left(x^{2}+y^{2}\right)+\frac{2 m_{1}^{\prime}}{\rho_{1}}+\frac{2 m_{2}}{\rho_{2}}+\frac{2 m_{3}}{\rho_{3}}-C .
$$

Let $r_{i}=\sqrt{\left(x-x_{i}\right)^{2}+\left(y-y_{i}\right)^{2}}$; then, if we suppose that the infinitesimal body moves in the $x y$-plane, the system of equations (6) reduces to the fourth order, and the integral becomes

$$
\left(\frac{d x}{d t}\right)^{2}+\left(\frac{d y}{d t}\right)^{2}=n^{2}\left(x^{2}+y^{2}\right)+\frac{2 m_{1}}{r_{1}}+\frac{2 m_{2}}{r_{2}}+\frac{2 m_{3}}{r_{3}}-C .
$$

If one integral more could be found under this last hypothesis, $J_{\text {ACOBI's theory }}$ of the last multiplier would be applicable, and the problem could be completely solved. However, this system of differential equations is of the type considered by Bruns in Acta Mathematica, vol. 11, for which he proved that there are no new algebraic integrals. It is equally of the type considered by Poincare in Les Méthodes Nouvelles de la Mécanique Céleste, vol. 1, chap. V, for which he proved that there are no new uniform transcendental integrals, even for very small relative masses of two of the three finite bodies.

§3. The Lagrangian solutions when the bodies revolve in circles.

Suppose first that the three bodies move so as always to lie in a straight line. Suppose the order of the bodies is $m_{1}, m_{2}, m_{3}$. Suppose the distances between $m_{1}$ and $m_{2}, m_{2}$ and $m_{3}, m_{3}$ and $m_{1}$ are respectively $1, a_{1}$ and $a_{2}$. Therefore $a_{2}=1+a_{1}$. Then the equation for the determination of $a_{1}$ is *

$$
\begin{aligned}
\left(m_{1}+m_{2}\right) a_{1}^{5}+\left(3 m_{1}+2 m_{2}\right) a_{1}^{4}+\left(3 m_{1}+m_{2}\right) a_{1}^{3}-\left(m_{2}+3 m_{3}\right) a_{1}^{2} \\
-\left(2 m_{2}+3 m_{3}\right) a_{1}-\left(m_{2}+m_{3}\right)=0 .
\end{aligned}
$$

* Laplace, Méc. Cél., vol. v, p. 310, or Tisserand, Méc. Cél., vol. I, p. 155. 
The $a_{1}$ to be chosen is the single real positive root of (9). Then the coördinates of the three bodies are given without ambiguity by

$$
\left\{\begin{array}{c}
m_{1} x_{1}+m_{2} x_{2}+m_{3} x_{3}=0 \\
x_{2}-x_{1}=1 \\
x_{3}-x_{2}=a_{1} \\
y_{1}=y_{2}=y_{3}=0
\end{array}\right.
$$

As there are three arbitrary units, of which only two have been thus far selected, the angular velocity may be chosen arbitrarily, and the unit of mass then determined. Or, if the unit of mass is taken arbitrarily, the angular velocity is given by the equation

$$
n^{2}=m_{1}+m_{2}+m_{3}\left(\frac{1}{a_{2}^{2}}-\frac{1}{a_{1}^{2}}\right) .
$$

There are two other different arrangements of the three bodies upon a straight line, in which their order is cyclically permuted. Their coördinates are determined in a manner similar to that employed above.

In the second Lagrangian solution the three bodies always lie at the vertices of an equilateral triangle. A side of the triangle being taken as the unit of length, the expression for the angular velocity is given by

$$
n^{2}=m_{1}+m_{2}+m_{3}
$$

Suppose that the axes are so chosen at the origin of time that $m_{1}$ is on the $x$-axis ; then the coördinates of the three bodies are determined by the equations

$$
\left\{\begin{array}{c}
y_{1}=0 \\
m_{1} x_{1}+m_{2} x_{2}+m_{3} x_{3}=0 \\
m_{1} y_{1}+m_{2} y_{2}+m_{3} y_{3}=0 \\
\left(x_{2}-x_{1}\right)^{2}+\left(y_{2}-y_{1}\right)^{2}=1 \\
\left(x_{3}-x_{2}\right)^{2}+\left(y_{3}-y_{2}\right)^{2}=1 \\
\left(x_{1}-x_{3}\right)^{2}+\left(y_{1}-y_{3}\right)^{2}=1
\end{array}\right.
$$

There is evidently but one distinct configuration of this type for three given bodies.

\$4. Surfaces of zero relative velocity in the case of the straight-line Lagrangian solutions.

The relative velocity of the infinitesimal body being represented by $V$, equation (7) may be written in this case 


$$
\begin{aligned}
V^{2}=n^{2}\left(x^{2}+y^{2}\right)+\frac{2 m_{1}}{\left\{\left(x-x_{1}\right)^{2}+y^{2}+z^{2}\right\}^{3 / 2}} & +\frac{2 m_{2}}{\left\{\left(x-x_{2}\right)^{2}+y^{2}+z^{2}\right\}^{3 / 2}} \\
& +\frac{2 m_{3}}{\left\{\left(x-x_{3}\right)^{2}+y^{2}+z^{2}\right\}^{3 / 2}}-C .
\end{aligned}
$$

This equation serves to give the relative velocity of the infinitesimal body at every point of rotating space; or, if the velocity be chosen arbitrarily, it gives the surfaces at which the infinitesimal body will move with the given velocity. The most interesting surfaces are those at which the velocity is zero, for they separate those portions of relative space in which the infinitesimal body may move from those in which it cannot. Setting $V$ equal to zero we have

$$
\begin{aligned}
n^{2}\left(x^{2}+y^{2}\right)+\frac{2 m_{1}}{\left\{\left(x-x_{1}\right)^{2}+y^{2}+z^{2}\right\}^{3 / 2}} & +\frac{2 m_{2}}{\left\{\left(x-x_{2}\right)^{2}+y^{2}+z^{2}\right\}^{3 / 2}} \\
& +\frac{2 m_{3}}{\left\{\left(x-x_{3}\right)^{2}+y^{2}+z^{2}\right\}^{3 / 2}}=C .
\end{aligned}
$$

The surfaces of which $(14)^{\prime}$ is the equation are symmetrical with respect to the $x y$ - and $x z$-planes, and a line parallel to the $z$-axis will pierce them in two (or no) points. Moreover, the surfaces are contained within a cylinder whose axis is the $z$-axis, and whose radius is $\sqrt{ } \bar{C} / n$, to which certain of the folds are asymptotic at $z^{2}=\infty$.

The intersection of the surfaces with the $x y$-plane is given by

$$
n^{2}\left(x^{2}+y^{2}\right)+\frac{2 m_{1}}{r_{1}}+\frac{2 m_{2}}{r_{2}}+\frac{2 m_{3}}{r_{3}}=C .
$$

For large values of $x$ and $y$ which satisfy this equation we have approximately

$$
n^{2}\left(x^{2}+y^{2}\right)=C \text {. }
$$

Therefore for sufficiently large values of $C$ one branch of the curves in the $x y$-plane is approximately a circle near the asymptotic cylinder.

For small values of $x$ and $y$ satisfying (15) we have

$$
\frac{m_{1}}{r_{1}}+\frac{m_{2}}{r_{2}}+\frac{m_{3}}{r_{3}}=\frac{C}{2} \text {. }
$$

This is the equation of the well-known equipotential curves for three centers of force, and for sufficiently large values of $C$ they consist of closed ovals around each of the bodies. For smaller values of $C$ these ovals coalesce by enlarging, while the large oval mentioned above diminishes in size.

The approximate equations of the curves in the $x z$-plane, for large and small values respectively of $x$ and $z$ are

$$
\begin{gathered}
n^{2} x^{2}=C, \\
\frac{m_{1}}{\left\{\left(x-x_{1}\right)^{2}+z^{2}\right\}^{3 / 2}}+\frac{m_{2}}{\left\{\left(x-x_{2}\right)^{2}+z^{2}\right\}^{3 / 2}}+\frac{m_{3}}{\left\{\left(x-x_{3}\right)^{2}+z^{2}\right\}^{3 / 2}}=\frac{C}{2} .
\end{gathered}
$$


The first is the equation of two straight lines parallel to the $z$-axis ; the second is the equation of equipotential curves. Therefore the locus in the xz-plane for sufficiently large values of $C$ is composed of curves asymptotic to the intersection of the asymptotic cylinder with the $x z$-plane and of ovals around each of the three bodies.

The approximate equations of the curves in $y z$-plane for large and small values respectively of the coördinates are

$$
\begin{gathered}
n^{2} y^{2}=C, \\
\frac{m_{1}}{\left(x_{1}^{2}+y^{2}+z^{2}\right)^{3 / 2}}+\frac{m_{2}}{\left(x_{2}^{2}+y^{2}+z^{2}\right)^{3 / 2}}+\frac{m_{3}}{\left(x_{3}^{2}+y^{2}+z^{2}\right)^{3 / 2}}=\frac{C}{2} .
\end{gathered}
$$

The first is the equation of two straight lines parallel to the $z$-axis. If the bodies lie in the order $m_{1}, m_{2}, m_{3}$, then $x_{2}$ will be much smaller than $x_{1}$ and $x_{3}$, unless there is a very great difference in the masses $m_{1}$ and $m_{3}$. If $m$ and $m_{3}$ are nearly equal the last equation becomes approximately

$$
\frac{m_{2}}{\left(x_{2}^{2}+y^{2}+z^{2}\right)^{3 / 2}}=\frac{C}{2},
$$

which is the equation of a circle. If $m_{1}$ is very much greater than $m_{3}$, then $x_{1}$ is small compared to $x_{2}$ and $x_{3}$, and we have approximately

$$
\frac{m_{1}}{\left(x_{1}^{2}+y^{2}+z^{2}\right)^{3 / 2}}=\frac{C}{2}
$$

which is also the equation of a circle.

Thus we conclude that for sufficiently large values of $C$, the surfaces are composed of a closed fold, roughly spherical in form, around each of the finite bodies, and curtains hanging from the asymptotic cylinder symmetrically with respect to the $x y$-plane; for smaller values of $C$, these folds and curtains unite; and for sufficiently small values of $C$ the surfaces are composed of two parts symmetrical with respect to the $x y$-plane but not intersecting it.

The motion of the infinitesimal body is real within the small folds around the finite bodies, and outside of the curtain hanging from the asymptotic cylinder, while it is imaginary in all the other portions of relative space. Therefore, if the infinitesimal body is within one of these closed regions at the origin of time, it will forever remain there.

Points on the surfaces may actually be computed most readily by first determining the curves in the $x y$-plane, and then finding, by methods of approximation, values of $z^{2}$ which will satisfy $(14)^{\prime}$. This is true since approximate values of $z^{2}$ are known from the general nature of the surfaces, when the curves in the $x y$-plane are given. 
If (15) is rationalized and cleared of fractions the resulting equation is of the 40 th degree in the variables $x$ and $y$, and may be used for the determination of points on the curves. The difficulty of locating the roots of an equation of so high degree is supplemented by the presence of foreign solutions which have been introduced in the process of rationalization. The latter difficulty can be avoided entirely and the degree of the equation notably reduced by transforming to bipolar coördinates.

If $m_{1}$ is supposed to lie in the negative direction from the origin on the $x$-axis, it is found without difficulty that

$$
x^{2}+y^{2}=x_{2} r_{1}^{2}-x_{1} r_{2}^{2}+x_{1} x_{2} .
$$

Then (15) becomes

$$
n^{2}\left(x_{2} r_{1}^{2}-x_{1} r_{2}^{2}\right)+\frac{2 m_{1}}{r_{1}}+\frac{2 m_{2}}{r_{2}}+\frac{2 m_{3}}{r_{3}}=C-n^{2} x_{1} x_{2} .
$$

$r_{3}^{2}$ can be expressed rationally in terms of $r_{1}$ and $r_{2}$ by an equation of the second degree. Therefore equation (17) may be written as a polynomial in $r_{1}$ and $r_{2}$ of the 10th degree. Points on the curves may be found by assuming convenient values of one of the variables and finding the real positive roots of the resulting equation, which will be of the 8 th degree in the other variable. The points of intersection of the circles around $m_{1}$ and $m_{2}$ as centers with the assumed and computed values respectively of $r_{1}$ and $r_{2}$ as radii, will be points of the locus.

\section{§5. Double points of the surfaces.}

It follows from the symmetrical forms of the surfaces that, as they degenerate and coalesce with decreasing values of $C$, all of the double points will appear in the $x y$-plane. Therefore the double points of the curves in the $x y$-plane alone may be sought. That is, the values of $C$ for which double points occur and their positions must be determined from the equation

$$
\begin{aligned}
n^{2}\left(x^{2}+y^{2}\right)+\frac{2 m_{1}}{\left\{\left(x-x_{1}\right)^{2}+y^{2}\right\}^{3 / 2}} & +\frac{2 m_{2}}{\left\{\left(x-x_{2}\right)^{2}+y^{2}\right\}^{1 / 3}} \\
& +\frac{2 m_{3}}{\left\{\left(x-x_{3}\right)^{2}+y^{2}\right\}^{1 / 4}}=C .
\end{aligned}
$$

The conditions for a double point are

$$
\left\{\begin{array}{l}
\frac{\partial C}{\partial x} \equiv n^{2} x-\frac{m_{1}\left(x-x_{1}\right)}{r_{1}^{3}}-\frac{m_{2}\left(x-x_{2}\right)}{r_{2}^{3}}-\frac{m_{3}\left(x-x_{3}\right)}{r_{3}^{3}}=0, \\
\frac{\partial C}{\partial y} \equiv n^{2} y-\frac{m_{1} y}{r_{1}^{3}}-\frac{m_{2} y}{r_{2}^{3}}-\frac{m_{3} y}{r_{3}^{3}}=0 .
\end{array}\right.
$$


There are certain double points on the $x$-axis. They are determined by the eqúation

$$
n^{2} x-\frac{m_{1}\left(x-x_{1}\right)}{\left\{\left(x-x_{1}\right)^{2}\right\}^{\frac{3}{2}}}-\frac{m_{2}\left(x-x_{2}\right)}{\left\{\left(x-x_{2}\right)^{2}\right\}^{\frac{3}{2}}}-\frac{m_{3}\left(x-x_{3}\right)}{\left\{\left(x-x_{3}\right)^{2}\right\}^{\frac{3}{2}}}=0 .
$$

The left side of this expression, considered as a function of $x$, changes sign once, and only once, in each of the intervals $+\infty>x>x_{3}, x_{3}>x>x_{2}, x_{2}>x$ $>x_{1}, x_{1}>x>-\infty$. Therefore there are four double points of the curves on the $x$-axis, one in each of the intervals mentioned. Expressing equation (20) in terms of $r_{3}$ as the variable and clearing of fractions, we have a rational equation of the 7 th degree in $r_{3}$. It has but one real positive root for the form which it takes in each of the intervals ; therefore it locates the positions of the double points, which are on the $x$-axis, uniquely. By substituting the coördinates of these various points in (18) the corresponding values of $C^{\gamma}$ are determined.

The double points for which $y$ is different from zero are found by solving (19) after dividing the second equation by $y$. By simple combinations of the two we find

$$
\left\{\begin{array}{l}
\text { (a) } n^{2} x_{3}-\frac{m_{1}\left(x_{3}-x_{1}\right)}{\left\{\left(x-x_{1}\right)^{2}+y^{2}\right\}^{\frac{3}{2}}}-\frac{m_{2}\left(x_{3}-x_{2}\right)}{\left\{\left(x-x_{2}\right)^{2}+y^{2}\right\}^{3}}=0, \\
\text { (b) } n^{2} x_{1}+\frac{m_{2}}{\left\{\left(x-x_{2}\right)^{2}+y^{2}\right\}^{\frac{3}{3}}}+\frac{m_{3}\left(x_{3}-x_{1}\right)}{\left\{\left(x-x_{3}\right)^{2}+y^{2}\right\}^{3}}=0, \\
\text { (c) } n^{2} x_{2}-\frac{m_{1}}{\left\{\left(x-x_{1}\right)^{2}+y^{2}\right\}^{\frac{3}{2}}}+\frac{m_{3}\left(x_{3}-x_{2}\right)}{\left\{\left(x-x_{3}\right)^{2}+y^{2}\right\}^{\frac{3}{3}}}=0 .
\end{array}\right.
$$

Any one of these three equations may be derived from the other two, and the simultaneous solution of any two of them will furnish all of the double points not on the $x$-axis.

The equations (19) are equally the necessary conditions for a minimum value of $C$. From (18) it is evident that $C$ has a minimum value, and it is easy to show that the sufficient conditions have not been fulfilled in the case of any of the solutions for which $y$ is zero, hence it is inferred that there is at least one solution of the system (21).

Suppose $m_{1}>m_{3}$; then $x_{1}<0, x_{2}>0$ and $x_{3}>x_{2}>0$. The equation (c) is not fulfilled for any value of $y^{2}$ when $x$ is greater than $x_{2}$; hence there is no solution in the interval $x_{2} \leqq x<+\infty$. Likewise $(b)$ is not fulfilled for any value of $y^{2}$ when $x$ is less than $x_{1}$; hence there is no solution in the interval $x_{1} \geqq x>-\infty$. Therefore the solutions must all lie in the interval $x_{1}<x<x_{2}$. For small values of $y^{2},(b)$ and $(c)$ are fulfilled by values of $x$ near $x_{1}$ and $x_{2}$ respectively. For increasing values of $y^{2}$ they are fulfilled by values of $x$ continuously increasing in distance from $x_{1}$ and $x_{2}$ respectively; 
therefore there is one, and only one solution in this interval. The sufficient conditions for a minimum value of $C$ are fulfilled in this case, and the curves in the $x y$-plane have degenerated to two points symmetrically situated with respect to the $x$-axis. There are, therefore, for one distribution of the finite bodies on a line, four double points on the $x$-axis and two situated symmetrically with respect to it.

In the special case in which $m_{1}=m_{3}$ the solution occurs for $x=x_{2}=0$, the $y$ coördinate being given by the equations

$$
\left\{\begin{array}{l}
n^{2}=\frac{m_{1}}{4}+m_{2}, \\
n^{2}=\frac{m_{2}}{\left\{y^{2}\right\}^{3}}-\frac{2 m_{1}}{\left\{1+y^{2}\right\}^{3}}=0 .
\end{array}\right.
$$

The $y$ coördinate has a maximum value for $m_{2}=0$, and decreases as $m_{2}$ increases.

\$6. Particular solutions of the motion of the infinitesimal body when the finite bodies move in circles according to the straight-line Lagrangian solution.

The surfaces of zero relative velocity discussed in $\$ 4$ are surfaces which the infinitesimal body cannot cross. Besides, it is easy to show that the lines of effective force are orthogonal to these surfaces. It follows that if the infinitesimal body is placed at a point in a surface of zero velocity it will move at first orthogonally to the surface. At the double points it seems that there would not be a tendency to move in any direction and it might be inferred that the body would remain relatively at rest.

The direct proof of this is that if the coördinates of the infinitesimal body fulfil the conditions for double points, (19), they also identically verify the differential equations of motion, (4).

There are thus, for each of the three permutations of the order of the finite bodies, four solutions of the problem in which the four bodies all lie in a straight ine, and two in which only the three finite bodies lie in a straight line. Therefore, for three given finite bodies and one of infinitesimal mass, there are eighteen, and no more, different solutions of arbitrary period of revolution, such that the finite bodies lie in a line while the mutual distances of the four bodies are constants.

§ 7. Surfaces of zero relative velocity in the equilateral triangular Lagrangian solution.

Suppose the three finite bodies revolve in circles so as to remain at the vertices of an equilateral triangle, and that the axes are chosen so that the $x$-axis 
passes through $m_{1}$. Then the equation of the surfaces of zero relative velocity is found from $(7)$ to be

$$
\begin{aligned}
n^{2}\left(x^{2}+y^{2}\right)+\frac{2 m_{1}}{\left\{\left(x-x_{1}\right)^{2}+y^{2}+z^{2}\right\}^{3 / 2}} & +\frac{2 m_{2}}{\left\{\left(x-x_{2}\right)^{2}+\left(y-y_{2}\right)^{2}+z^{2}\right\}^{3 / 2}} \\
& +\frac{2 m_{3}}{\left\{\left(x-x_{3}\right)^{2}+\left(y-y_{3}\right)^{2}+z^{2}\right\}^{3 / 6}}=C .
\end{aligned}
$$

The surfaces are symmetrical with respect to the $x y$-plane, and are enclosed within a cylinder whose axis is the $z$-axis and whose radius is $\sqrt{C} / n$, and to which certain folds are asymptotic at $z^{2}=\infty$.

The equation of the trace of the surfaces upon the $x y$-plane is

$$
\begin{aligned}
n^{2}\left(x^{2}+y^{2}\right)+\frac{2 m_{1}}{\left\{\left(x-x_{1}\right)^{2}+y^{2}\right\}^{3 / 2}}+ & \frac{2 m_{2}}{\left\{\left(x-x_{2}\right)^{2}+\left(y-y_{2}\right)^{2}\right\}^{1 / 2}} \\
& +\frac{2 m_{3}}{\left\{\left(x-x_{3}\right)^{2}+\left(y-y_{3}\right)^{2}\right\}^{3 / 2}}=C .
\end{aligned}
$$

For respectively large and small values of $x$ and $y$ satisfying this equation we have approximately

$$
\begin{gathered}
n^{2}\left(x^{2}+y^{2}\right)=C, \\
\frac{2 m_{1}}{\left\{\left(x-x_{1}\right)^{2}+y^{2}\right\}^{3 / 2}}+\frac{2 m_{2}}{\left\{\left(x-x_{2}\right)^{2}+\left(y-y_{2}\right)^{2}\right\}^{3 / 2}}+\frac{2 m_{3}}{\left\{\left(x-x_{3}\right)^{2}+\left(y-y_{3}\right)^{2}\right\}^{3 / 6}}=C .
\end{gathered}
$$

The first equation shows that, for sufficiently large values of $C$, one branch of the curves is approximately a circle lying near the asymptotic cylinder. The second is the equation of the equipotential curves, which, for sufficiently large values of $C$, consist of closed ovals around each of the three finite bodies. For smaller values of $C$ these smaller ovals coalesce by enlarging, while the large oval unites with the smaller ones by decreasing in size.

The trace of the surfaces in the $x z$-plane is composed of two curves asymptotic to the lines of intersection of the plane and the asymptotic cylinder, together with an oval around $m_{1}$. If the $x$-axis had been directed so as to pass through either of the other two finite bodies similar results would have been true. Therefore, we conclude that, for sufficiently large values of $C$, the surfaces are composed of curtains hanging from the asymptotic cylinder, and closed folds approximately spherical in form around each of the finite bodies.

The motion of the infinitesimal body is real within the closed folds and outside of the curtains. If the infinitesimal body were within one of these closed regions at the origin of time it would forever remain there.

In order actually to compute points on the surfaces, it is advantageous to 
transform the equation of the curves in the $x y$-plane to bipolar coördinates. Points on the surfaces can then be rapidly found by methods of approximation.

\section{§8. Double points of the surfaces.}

It follows from the symmetry of the surfaces that the double points which appear as $C$ decreases will all lie in the $x y$-plane. They will evidently appear, (a) where the small ovals around the finite bodies first touch each other; (b) where the small ovals first touch the large oval; $(c)$ where the small triangular curves, whose sides are formed from two of the small ovals and the large one, vanish from the $x y$-plane; and $(d)$ where the triangular curves, whose sides are formed from the three small ovals when they unite, vanish from the $x y$-plane. The points $(c)$ and $(d)$ occur for minima of $C$ as defined by (24), the absolute minimum depending upon the distribution of the masses in the finite bodies.

The conditions for double points are found from (2t) to be

$$
\left\{\begin{array}{l}
\frac{\partial C}{\partial x} \equiv n^{2} x-\frac{m_{1}\left(x-x_{1}\right)}{r_{1}^{3}}-\frac{m_{2}\left(x-x_{2}\right)}{r_{2}^{3}}-\frac{m_{3}\left(x-x_{3}\right)}{r_{3}^{3}}=0 \\
\frac{\partial C}{\partial y} \equiv n^{2} y-\frac{m_{1} y}{r_{1}^{3}}-\frac{m_{2}\left(y-y_{2}\right)}{r_{2}^{3}}-\frac{m_{3}\left(y-y_{3}\right)}{r_{3}^{3}}=0 .
\end{array}\right.
$$

In order to exhibit most clearly the distribution of the double points and omitting to give the direct proofs from the equations, which would be similar to those given in the former case, let us solve numerically the simple example in which $m_{1}=m_{2}=m_{3}=1$. Then $n^{2}=3$. Since the figure is symmetrical with respect to the three axes passing through the center of gravity and the three bodies respectively, and since all the double points lie on these axes, it will be sufficient to locate the double points on one of them. In this case the second of (25) is fulfilled for $y=0$, and the first becomes, a side of the triangle being taken as unit of length,

$$
3 x-\frac{\left(x-\frac{1}{\sqrt{3}}\right)}{\left\{\left(x-\frac{1}{\sqrt{3}}\right)\right\}^{3}}-\frac{2\left(x+\frac{1}{2 \sqrt{3}}\right)}{\left\{\left(x+\frac{1}{2 \sqrt{3}}\right)^{2}+\frac{1}{4}\right\}^{3}}=0 .
$$

The real solutions of this equation are approximately $x=1.22, x=0, x=-.24$ and $x=-.93$. The first belongs to the class $(b)$ defined at the beginning of this section, the second to the class $(d)$, the third to the class $(a)$, and the fourth to the class $(c)$. There are corresponding solutions on the other two axes, and these are the only real solutions of the equations. As the point $x=0$ has been counted three times it is seen that there are ten double points of the surfaces 
of zero relative velocity in the case of the triangular solution of the problem of three bodies.

\$9. Particular solutions of the motion of the infinitesimal body in case the finite bodies move in circles according to the equilateral triangular Lagrangian solution.

By general considerations similar to those advanced in $\S 6$ we conclude that the double points are points of zero effective force, and that if the infinitesimal body is placed at one of them with zero relative velocity it will always remain in the same relative position.

The direct proof that these are particular solutions of the problem is that if the relative coördinates of the infinitesimal body are supposed to be constants fulfilling the conditions for double points (25), they also verify identically the differential equations of motion (4).

Therefore, three finite bodies of arbitrary masses and one infinitesimal body may be started in twenty-eight, and no more, different ways so that they will revolve in an arbitrary period and preserve their mutual distances.

The University of Chicago. 